\title{
Distribution of Materials in Road Earthmoving and Paving: Mathematical Programming Approach
}

\author{
Roberto Xavier de Lima ${ }^{1}$; Ernesto Ferreira Nobre Júnior ${ }^{2}$; Bruno de Athayde Prata ${ }^{3}$; and José Weissmann ${ }^{4}$
}

\begin{abstract}
This paper summarizes a mathematical model that relates the geometric and geotechnical features of a road construction site with the allocation of materials, searching for a minimum construction cost. This paper proposes a linear programming model to optimize excavation and paving services. With this model, it is possible to evaluate site alternatives with different soil strata and different degrees of compaction. The borrow pit materials are allocated in the most economical way, and it is possible to incorporate more inputs like materials mix. Software was used to solve the model, and a spreadsheet application was used as an interface for data input. The proposed model demonstrated possible cost savings in earthwork planning. It is expected that earthwork and paving optimization with linear programming will reduce road construction costs considerably. DOI: 10.1061/(ASCE)CO.1943-7862.0000666. (c) 2013 American Society of Civil Engineers.
\end{abstract}

CE Database subject headings: Highway engineering; Earthmoving; Logistics; Computer programming; Construction materials; Mathematical models.

Author keywords: Highway engineering; Earthmoving logistics; Mixed integer linear programming.

\section{Introduction}

Earthmoving and paving are the most expensive items in the composition of the total cost of road construction. These costs are incurred due to the transportation of large quantities of soil, since these materials often have to travel thousands of miles to the point of placement. As the heavy construction industry demanded more efficient equipment, mechanized construction experienced a dramatic increase in productivity in the last century, replacing manual work once and for all.

Ricardo and Catalani (2007) state that mechanization requires large investments in expensive equipment, combined with rationally planned and well-executed construction work. Consequently, the allocation of resources should be wisely considered so that the costs invested to perform the work are minimized. Therefore, for optimum road construction planning, it is necessary to accurately identify the destination and the amount of materials coming from

\footnotetext{
${ }^{1}$ Senior Engineer, Dept. of Highways of Ceará, Brazil; and Researcher, Dept. of Transportation Engineering, Research Group on Logistics and Network Infrastructure, Federal Univ. of Ceará, 60020-181, Fortaleza, Ceará, Brazil. Email: rxdelima@yahoo.com.br

${ }^{2}$ Associate Professor, Dept. of Transportation Engineering; Member of Doctoral Program in Transportation Engineering, Research Group on Logistics and Network Infrastructure, Federal Univ. of Ceará, 60020181, Fortaleza, Ceará, Brazil; and Vice President, Brazilian Institute of Audit Engineering. Email: nobre@ufc.br

${ }^{3}$ Adjunct Professor, Center of Technology, Research Group on Logistics and Network Infrastructure, Federal Univ. of Ceará, 60020-181, Fortaleza, Ceará, Brazil (corresponding author). E-mail: baprata@ufc.br

${ }^{4}$ Professor, Dept. of Civil and Environmental Engineering, Master Program Coordinator, Univ. of Texas, San Antonio, TX 78249; and Research Group on Logistics and Network Infrastructure, Federal Univ. of Ceará, 60020-181, Fortaleza, Ceará, Brazil. Email: jose.weissmann@utsa.edu

Note. This manuscript was submitted on May 25, 2012; approved on December 12, 2012; published online on December 14, 2012. Discussion period open until January 1, 2014; separate discussions must be submitted for individual papers. This paper is part of the Journal of Construction Engineering and Management, Vol. 139, No. 8, August 1, 2013. (C) ASCE, ISSN 0733-9364/(9)/\$25.00.
}

the various cut sections and also where these materials will be disposed of afterward.

This paper is based on a study that developed a mathematical model in which the geometric and geotechnical data of a road project is used to optimize the allocation of materials involved in earthwork and paving. The main objective of this paper is to present a model based on mixed linear programming techniques in order to optimize earthmoving and paving by seeking a solution that improves the distribution of materials at a minimal cost.

The proposed model analyzes earthmoving road construction tasks with different scenarios of soil strata and embankments and with varying degrees of compaction. The model also analyzes construction sites with nonlinear characteristics, such as urban sections or large areas of earthmoving.

For the paving component, the model optimizes the material storage points along the proposed roadway alignment as cost effectively as possible, while minimizing the total cost of the construction work. The model allows for two different types of analysis: one covers the cases of construction and restoration, the other covers cases of mixed soils in manufacturing plants and at the construction site.

When applied to real construction site problems, the alternatives for paving lead to hundreds and even thousands of combinations. The software tool documented in this paper provides a systematic way in which a user can solve a large volume of combinations leading to optimized solutions.

The proposed approach consists of a new mathematical model that incorporates a mix of materials in paving plants and their distribution to the respective pavement strata along the proposed roadway alignment. The ability to employ a model that accepts mixtures of soil, executed at the roadside and plants, opens up a wider range of alternative solutions. The use of different soil combinations in optimization models is not available in the existing literature, considering that not even the model developed by Jayawardarne (1994a, b) covers this alternative.

For the solution of the mixed integer programming problem (MIPP), the authors used the solver LINGO 6. To develop the user interface, Visual Basic for Applications (VBA) and Microsoft Excel were combined. This interface enables professionals-who 
do not master mathematical programming concepts- to use this optimization environment.

\section{Literature Review}

Earthwork represents the largest part of the total cost in road construction. Earthmoving and paving require special attention from contractors, given that these activities deal with the transportation of thousands of tons of material and a large number of heavy equipment pieces. Therefore, it is necessary that the transportation of materials between cut and fill sections and quarries be done in a rational manner, with the objective of attaining the lowest construction cost.

Mayer and Stark (1981) first studied these issues, using the term earthworks logistics. Earthworks logistics is an application of the general logistics to the particular case of earthmoving and paving roads. In this reference, there is a strong connection between material flows in the industrial sector and earthmoving.

The method of using a chart diagram of masses is the most commonly used process in the distribution of earthmoving material and equipment. This process is widely used in the construction industry to define the quantities of material to be transported and their destination. In essence, the method consists of a graphical representation of earth volumes indicating how to perform tradeoffs between cut and fill sections.

However, the mass diagram cannot be applied to nonlinear road works, such as urban areas or large areas of earthmoving. This model is also not suitable for paving operations using mixed soils, whether these soils are mixed in manufacturing plants or at the construction site.

According to Mayer and Stark (1981), the mass diagram is also not suitable for other situations, i.e., (1) when transport costs are not directly proportional to the distances, (2) soil characteristics vary over the proposed roadway alignment, (3) additional quantities of materials must be transported away from the local section, and (4) the purge or waste material must be removed from the alignment.

With the objective of overcoming some of the limitations of the mass diagram, linear programming models-first suggested by Stark and Nicholls (1972) and later developed by Mayer and Stark (1981), Nandgaonkar (1981), Easa (1987, 1988, 1989), Jayawardane and Price (1994a, b), Son et al. (2005), Moselhi and Alshibani (2009), and Hare et al. (2011)—may be applied.

The model developed by Mayer and Stark (1981) incorporates details such as embankment factors, allocation of deposit materials, materials with different strata, and with different degrees of compaction. By using continuous variables, the model developed by Mayer and Stark (1981) minimizes the costs of earthmoving. However, the authors did not make a distinction between earthmoving and paving. Later, Easa (1987) included variable unit costs to the model.

Easa (1988) presents a quadratic programming model for allocation of materials in earthmoving, which complemented the approaches based on mathematical programming. After further investigation, Easa (1988) found that the unit cost of earthmoving could be assumed to be constant.

Christian and Caldera (1988) presented a model for optimizing earthmoving operations based on a standard transportation problem. These authors consider (1) deposits in areas of cut and fill for the case of any differences between the amounts of cutting and filling, and (2) a high unit cost of transportation if the earthmoving is physically impossible between two points. Easa (1989) discussed the model developed by Christian and Caldera (1988), correcting some inconsistencies.
Using a quite complex model, Jayawardane and Price (1994a, b) introduced the possibility of taking the performance of equipment operations and the duration of the work into account, but did not cover the use of paving mixes and the allocation of paving plants.

Son et al. (2005) presented a linear programming model to determine the amount of soil to be transported, the shortest transport distances, and locations of deposits of cut and fill to be used in earthmoving.

Moselhi and Alshibani (2009) presented an approach for optimizing earthmoving operations in heavy civil engineering works. The proposed approach consists of several factors, such as resources available to contractors, time and/or budget constraints, characteristics of the project and the soil, indirect costs, and equipment features. The proposed approach uses genetic algorithms, linear programming, and geographic information systems in order to support the process of decision making.

Hare et al. (2011) proposed a MIPP that incorporates the traditional modeling (which seeks to minimize the material handling) to the existence of physical blocks. By the term "block," one should understand: rivers, group of trees, topographical issues, and so on. The incorporation of physical blocks makes the model considerably more difficult to be solved. Thus, strategies for reduction of computational time are required.

Based on the literature review, the shortcomings of the established modeling approaches were identified and served as a basis for the proposed improvements incorporated into the MIPP approach documented by this paper.

The innovations associated with the approach suggested by the authors may be subdivided into the following areas: earthmoving and paving, plant materials location, and materials blending at the roadside.

For earthmoving and paving, the model optimizes the distribution of materials following the longitudinal profile, with materials being distributed in layers as detailed by Figs. 1 and 2. The materials to be distributed may originate from the longitudinal profile, as well as from remote sites needed to supplement potential lack of materials from the cut sections. Consequently, the proposed model addresses the issues related to earthmoving and the issues related to the distribution of materials within the pavement layers.

The proposed model incorporates the location of the plants for materials mixing - an approach that was not available in the literature review. The model allows the user to evaluate the options for location of the materials mixing plants using as criteria the material transportation distances and the composition of the required materials to build the pavement base and subbase, such as the percentages of crushed stone and soil.

With the definition of the mix design for the different pavement layers and the optimized location of the materials mixing plants, the model establishes the optimized allocation of the materials sourced from the borrow sites and rock quarries.

In the proposed approach, the materials' blending was addressed in two ways. In addition to the process of optimizing the location of the materials mixing plants described previously, the model also addresses the possibility of mixing materials by the roadside. For the materials mixed at the roadside, the materials are supplied by rock quarries and borrow sites and are mixed by equipment at the roadside in the quantities established by the roadway design.

\section{Proposed Approach}

The use of a mix of manufactured materials is a solution often used in road paving. This may be caused by the unavailability of materials that meet the specifications or due to costs. 
The mix of materials of different textures and consistencies may result in a single material that meets the requirements of cohesion, plasticity, and strength. The dosage of these materials can be done by different methods that take into account their geotechnical characteristics such as granulometry, plasticity index, specific weight, and the limits required by specifications.

The model reported in this paper makes use of mixed materials with the assumption that both the ratios among them and the deposits that supply them are known beforehand and used as inputs to the model.

For the construction of a typical section of road work, it is understood that the following information is available: horizontal and longitudinal alignment; survey data; volume and location of cuts corresponding to different types of soils; volume and location of fills; cross sections with the thicknesses of the various layers of the pavement defined; location and volumes of deposits of materials to be used in the pavement cross section and embankments; location and capacity of areas for disposal of unused materials; density (specific weight) in situ of deposits; density (specific weight) on the as-built road (after compaction) of the various layers of the pavement; factors of shrink/swell of the material of the sections; definition of mixtures (deposits and proportions) to be used in layers of paving, whether performed on the roadside or in manufacturing plants; alternative manufacturing plant location for mixture of soils; and miscellaneous costs associated with the road work.

These data are schematically summarized by Figs. 1 and 2 .

During the development of the model, two types of variables were defined: earthmoving and paving. There are two reasons why the authors separated these types of variables: (1) to clarify the problem from the construction point of view, since they are conceptually two different types of activities; and (2) to reduce the number of constraints to be introduced in the model, given that the prior knowledge of the materials to be used in paving and earthwork reduces the need for adding new constraints. This distinction prevents, for example, the material of a given cut from being allocated to a pavement base, or a mixture of crushed soil from being allocated to an embankment.

Since the linear programming model of each deposit or cut is represented by a constraint on their capacity, a question is raised: is it possible to build two separate models, one for earthmoving and another for paving? The answer is yes, provided there are no deposits common to the two types of work and that no cutting material is used in paving. The authors also define that the amount of the material used in earthmoving and paving of each cut deposit should be less than the available quantity of each one of them.

The following MIPP is therefore defined, where the variables are explained in the notation list:

$$
\begin{aligned}
\text { Minimize } Z= & \sum_{s=1}^{s f} \sum_{c a=1}^{c a f} C S(s, c a) X S(s, c a) \\
& +\sum_{k=1}^{k f} \sum_{s=1}^{s f} C D(k, s) X D(k, s) \\
& +\sum_{i=1}^{n} \sum_{c a=1}^{c a f} C B(i, c a) X B(i, c a) \\
& +\sum_{w=1}^{w f} \sum_{p=1}^{p f} \sum_{j=1}^{m} C Y(w, p, j) Y(w, p, j) \\
& +\sum_{w p=1}^{w p f} \sum_{j=1}^{m} C P X(w p, j) X(w p, j)
\end{aligned}
$$

subject to
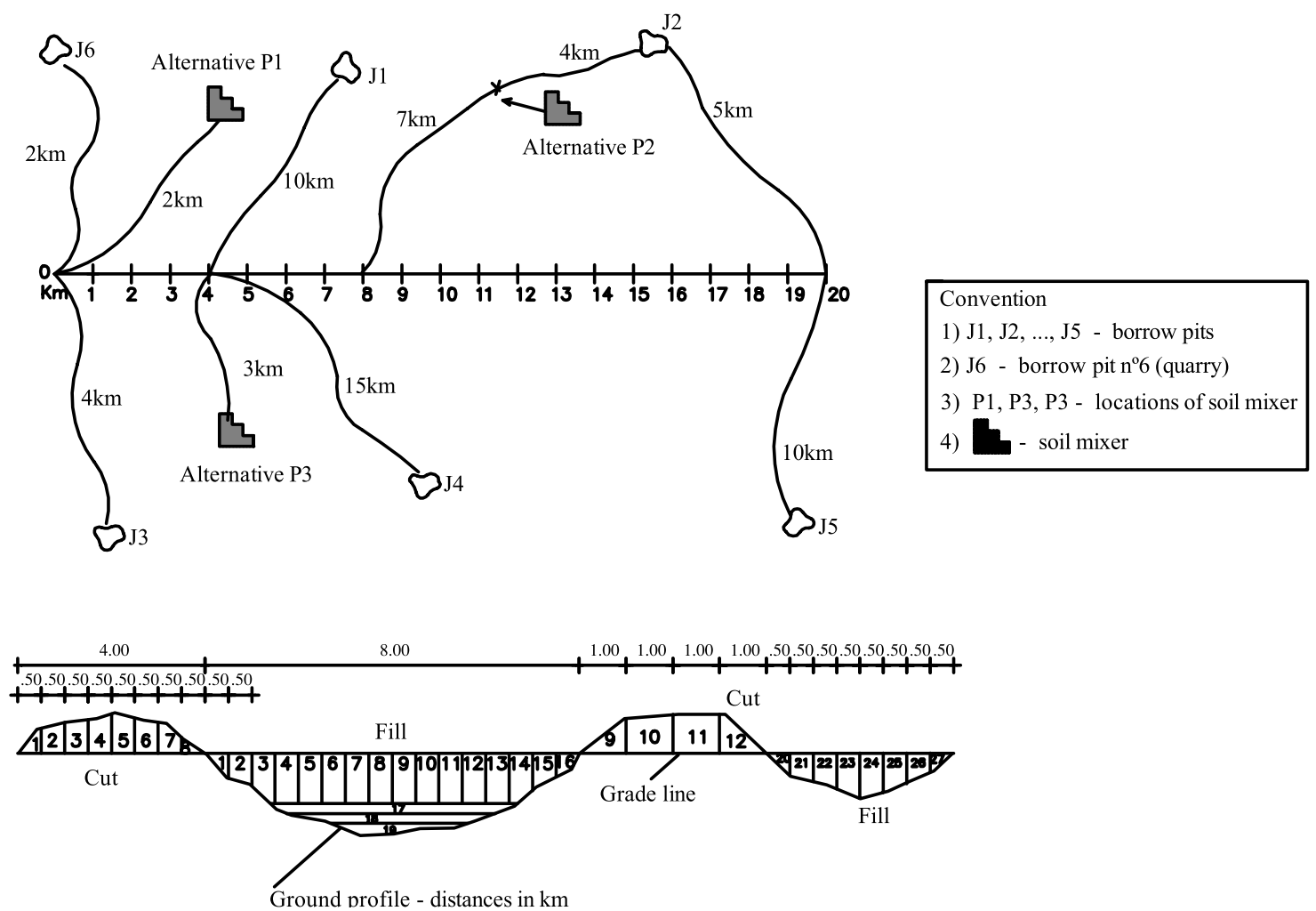

Fig. 1. Plan with location of mines, manufacturing plants, and profile with grade-numbered segments 


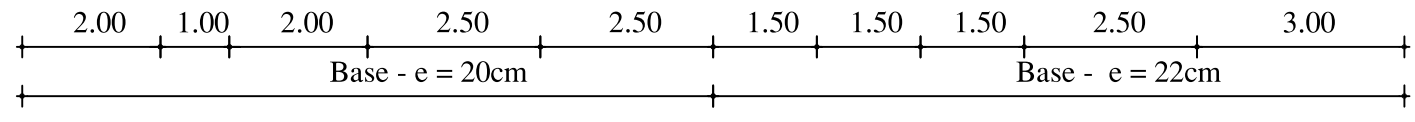

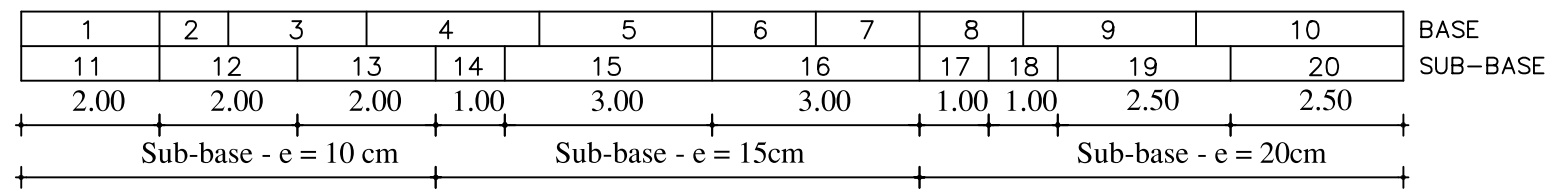

Pavement section - extensions in $\mathrm{km}$

Fig. 2. Profile and numbering of the pavement layers

$$
\begin{gathered}
\sum_{c a=1}^{c a f} X S(s, c a)+\sum_{k=1}^{k f} X D(k, s) \\
+\sum_{w p=1}^{w p f} \sum_{j=1}^{m} C R P X S(s, w p, j) X(w p, j) \\
+\sum_{w=1}^{w f} \sum_{p=1}^{p f} \sum_{j=1}^{m} C R Y S(s, w, p, j) Y(w, p, j)=V C(s) \\
\sum_{c a=1}^{c a f} X B(i, c a)+\sum_{w=1}^{w f} \sum_{p=1}^{p f} \sum_{j=1}^{m} C R Y(i, w, p, j) Y(w, p, j) \\
+\sum_{w p=1}^{w p f} \sum_{j=1}^{m} C R P X(i, w p, j) X(w p, j) \leq \mathrm{VOL}(i) \\
\sum_{s=1} X S(s, c a) F S(s, c a)+\sum_{i=1}^{n} X B(i, c a) F I(i, c a)=V A(c a) \\
\sum_{w=1}^{w f} \sum_{p=1}^{p f} Y(w, p, j)+\sum_{w p=1} \sum^{m} X D(k, s) F K C(k, s) \leq V B F(k)
\end{gathered}
$$

Eq. (1) is the objective function to be minimized, with the first three terms related to the cost of earthmoving and the last two to the cost of paving. The purpose of the model is to minimize the cost of road construction.

The sum of the $X S(s, c a)$ e $X D(k, s)$ variables for all the cutting strata represents the necessary amount of a cut for achieving the grade. The purging of material might occur for three reasons: the material may be unsuitable for embankments-for instance, rocky or organic materials - or even the quantity of material can be excessive; it may be necessary to purge a portion out; or, in certain situations, it may be more economical to import material from deposits, purging the excess. It is also assumed that the model areas for disposal of materials have been previously studied for the availability of capacities and locations. Similarly, the sum of the variables $X B(i, c a)$ for all strata represents the volume required of embankment for achieving the road grade.

The paving variables were also divided into mixes of variables processed at the roadside and plant manufactured mixtures. Thus, should a mix happen on the roadside, the model allocates each previously studied mix in the fitting segment in which pavement strata were subdivided. For manufactured mixes, the model identifies the description of mixes and chooses the best position of the manufacturing plant among the possible alternatives.

The reader should note that the numbering of the pavement strata is independent of the numbering of sections of earthwork. In earthwork each cut segment is divided into smaller portions, where materials are separated into first, second, and third categories. Materials that are unsuitable for use should also have separate numbering. For the embankments, the numbering may be done according to the degree of compaction of the strata, these being subdivided into segments, since they are very long.

In the case of variable paving, the numbering is established by subdividing the reinforcement strata of the subgrade, subbase, or base. The subdivision in intervals makes the determination of distances possible. However, this is not always possible, since rehabilitation work or segments composed by many urban areas or watercourses may result in naturally irregular subdivisions.

The group of equations of type (2) sets conditions for each section $s$. Each type of constraint (2) can be divided into four portions, described as follows: The first portion corresponds to the volume of material in $\mathrm{m}^{3}$, coming from the $s$ cut layer destined for the $c a$ embankment layer. The second part corresponds to the volume of material in $\mathrm{m}^{3}$, purged from the $s$ cut layer. The third part corresponds to the volume of material in $\mathrm{m}^{3}$ coming from the cut intended to make the $w p$, mixed on the road. The fourth part is the amount of material in $\mathrm{m}^{3}$ coming from the cut intended to make the manufactured mixture $w$.

In summary, the group of equations of type (2) imposes that the volume utilized from the cut layer $s$ must be equal to the total volume of the layer. The group of equations of type (3) sets conditions for each field in $i$. Each constraint type (3) can be divided into three parts, described as follows: The first part corresponds to the volume of material in $\mathrm{m}^{3}$ coming from the reservoir $i$ and destined to the $c a$ embankment layer. The second part corresponds to the volume of material in $\mathrm{m}^{3}$ coming from the reservoir $i$ and intended to compose the manufactured mixture $w$. The third part corresponds to the volume of material in $\mathrm{m}^{3}$ coming from the reservoir $i$ and intended to compose the $w p$, mixed on the roadside.

The group of equations of type (4) sets the conditions for each ca layer embankment. Each type of constraint (4) can be divided into two parts described as follows: The first part corresponds to the volume (geometric) in $\mathrm{m}^{3}$ from the $c a$ layer embankment formed by the material coming from the $s$ cutting layer. The second part corresponds to the volume (geometric) in $\mathrm{m}^{3}$ from the $c a$ embankment layer formed by material from the deposit $i$. The constraints of the type (4) require for each $c a$ embankment layer to be the sum of volumes (geometric) of the material of the cut and deposits to be equal to the total volume of the layer.

The group of equations of type (5) provides constraints to be met for each area reserved to disposal $k$. Each type of restriction (5) requires that the sum of volumes purged of the various strata of 
cuttings and destined for the disposal $k$ should be less than or equal to the capacity of the area. The group of constraints of type (6) provides that for each segment in which the strata of paving were subdivided, a only solution must be allocated.

Through the variables, the distribution model allocates earthwork and paving quantities of materials from the deposits or manufacturing sites in predetermined locations of the section. If materials of all deposits or mixtures are suitable for all segments, no additional constraint is needed. However, if a mixture is suitable for a segment, the variables of this mixture should be zero. Assuming that the segments $1-10$ are based on a given piece and $w=2$ is a mixture composed only of material of a subbase reservoir, then one must make $\mathrm{X}(2, j)=0$, for $j=1,2, \ldots, 10$.

In real-life applications, other situations may occur and some subjective judgment may be needed. If any cut material is unusable ( $s=1$, for example), one must make $\mathrm{X}(1, c a)=0$, thus preventing the material from being allocated in an embankment.

In exceptional situations, it is necessary to verify the operational feasibility of the solution. Take, for instance, the case where a large cut section is suitable as paving material. Here it is necessary to evaluate the impacts that this solution will have regarding excavation and transportation costs, since a paving section may be under the cut. This would make infeasible the use of cut for this section, or the removal, transportation, storage, and return transportation to the pavement section would have to be considered in the costs.

Finally, additional constraints may be used as a resource for reducing the number of variables. Consider those variables related to deposits that are far from the respective segments equal to zero. Nevertheless, this feature should be used with caution because, due to lack of material, its excessive use might render the problem mathematically unfeasible.

In the proposed model, it is assumed that the proportions of the materials originating from the borrow sites is known by the model user. This assumption deserves further discussion in this paper on how it impacts real-life applications of the model.

The standard practice in roadway construction planning is to identify borrow sites along the proposed roadway alignment. If these borrow sites do not meet the required specifications by the design, tests are performed by mixing borrow site materials or borrow site materials and industrialized materials. After these initial studies are completed, the final mix designs to be used in the project are defined and the information is available to the modeling process.

Another possibility is that during the actual construction procedures, some of these borrow sites become unavailable. In this case, new tests need to be performed to determine new material blending combinations. The resulting combinations have to be updated and the model rerun to determine a new set of solutions.

The model considers in its objective function the overall cost as a variable to be minimized. The unit costs driving the overall cost are related to materials, labor, and equipment.

Another set of pertinent comments about the model are related to the flexibility of the model relative to the variability of the situations faced at the construction site by a construction planner. As an example, the model does not currently address projects that involve recycling of materials at the project site. However, this option can be incorporated in future versions of the model. In addition, the model assumes that there are no outside factors interfering with the development of the sequence of tasks or in the order of the execution of these tasks. However, the model is generic enough to allow for its application to the majority of roadway construction projects.

\section{Case Studies}

\section{Methodology Applied in Case Studies}

One of the factors that limit the use of mathematical programming models in the area of road construction is the need for professionals who are knowledgeable in the area of operations research (OR).

In this context, one of the contributions of the approach proposed in this paper is the development of procedures which aid in the use of OR modeling in the construction industry by building user-friendly computerized interfaces.

The input data for the proposed mathematical programming model is accomplished through an Excel spreadsheet. With the help of the programming language VBA, it was possible to implement a routine for using Excel for generating the inputs for the optimization model LINGO. The Excel VBA model then runs LINGO to obtain the optimal solution. After the analysis run is completed, the results are exported to Excel, enabling analysis by industry professionals with no OR experience.

Fig. 3 depicts the integration of Excel and LINGO.

In order to use the proposed model, the authors adopted the following order: entry of geotechnical data and geometric costs in Excel; calculation of the coefficients using a routine written in VBA in Excel; reading the coefficients through a dynamic link library (DLL) available in LINGO; solution of the MIPP using the submitted script; generating the report file (.LOG) with the results in the LINGO standard; and generation of MIPP file (.TXT) in the $L I N G O$ standard with the coefficients calculated, and piping the results to Excel.

For the solution of the model for each case study, the authors followed the script below:

- Dividing and numbering of the pavement strata;

- Numbering the deposits, mixtures, and positions of the manufacturing plants;

- Determining the distances between cut, embankments, pavement strata, deposits, positions, and location of the manufacturing plants to disposal areas;

- Estimating the unit costs;

- Filling in Excel spreadsheets generated by the routine written in VBA;

- Calculating the coefficients of the variables from the data of the spreadsheets with the same routine. The calculated coefficients are automatically read by $L I N G O$;

- Solving the model using LINGO.

Fig. 4 depicts a flowchart of the proposed methodology.

The following data are required for the formulation of the mathematical model:

- Data for each segment: Number of borrow sites, number of segments, number of mixing plants, and required as-built densities;

- Borrow sites: material composition, volumes, and densities;

- Segment data: volumes of each section;

- Distances between segments, borrow sites, and mixing plants;

- Transportation costs between borrow sites and segments, costs of establishing the borrow sites, and costs of mixing.

The first step that the user has to accomplish is to divide and number the segments based on earthmoving and paving activities and input this information into the Excel spreadsheet.

The more refined (larger number of) the segments, the more precise the model solution, with an associated increase in data input labor. However, the division into small segments may increase the difficulty of implementation of the recommended operations at the construction site and decrease construction productivity. Based on the authors' experience, the suggested segment length 


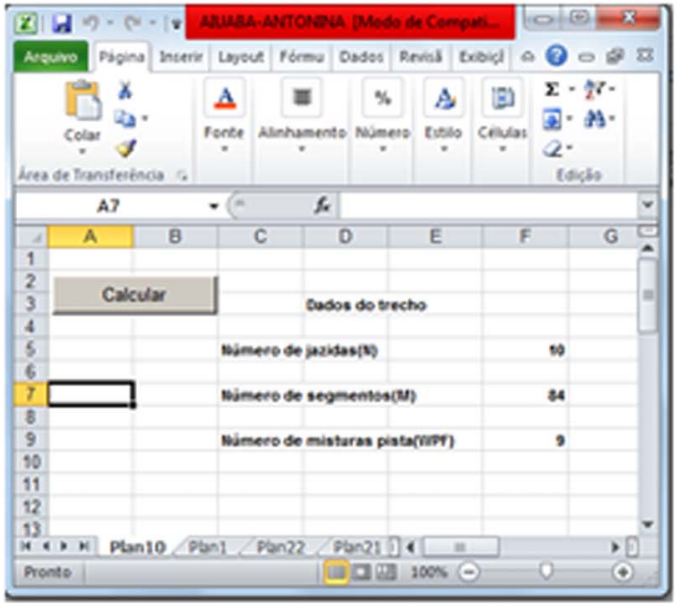

Excel/VBA - input

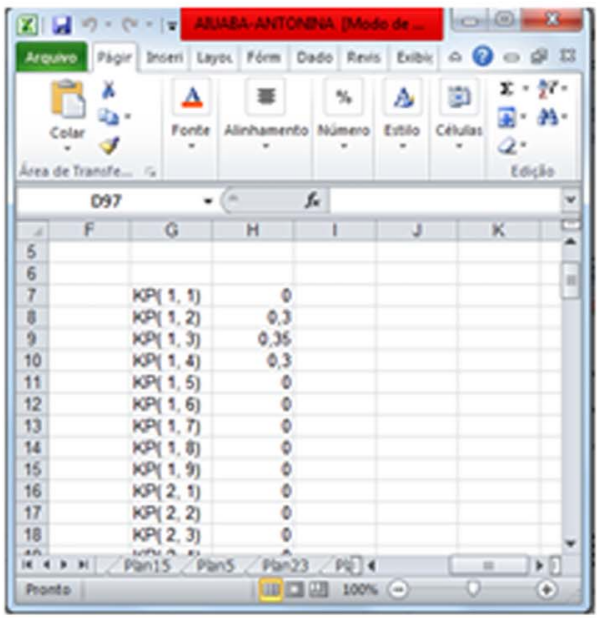

Excel/VBA - output

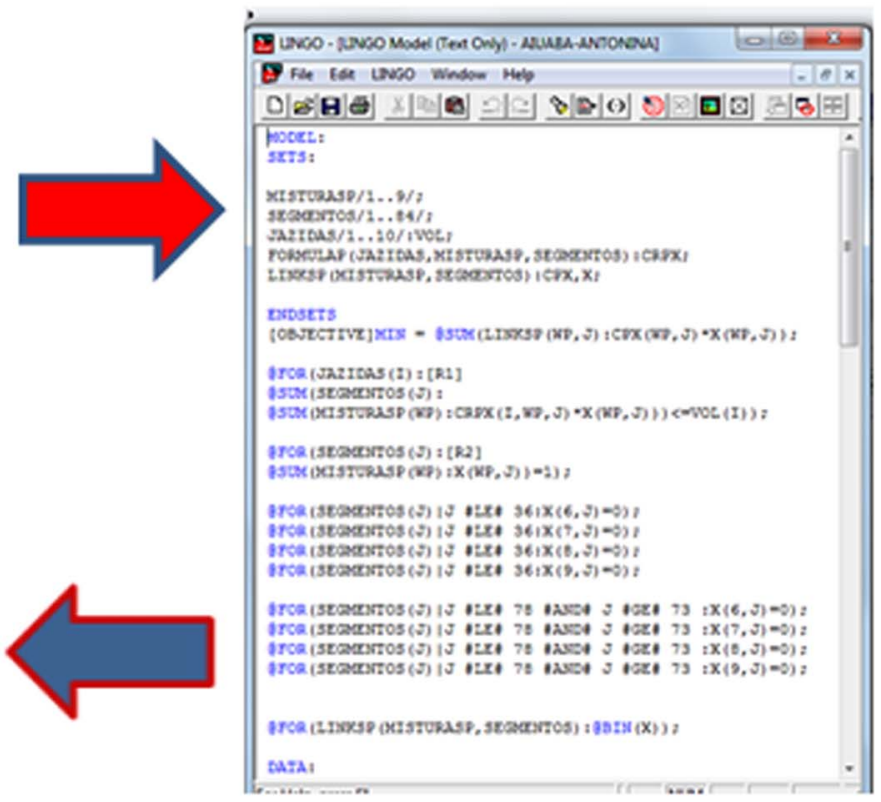

LINGO - optimization solver

Fig. 3. Interface between Excel and $L I N G O$

should be $500 \mathrm{~m}$. For more complex rehabilitation projects, a sensitivity analysis is recommended.

In the next step, the user inputs in the Excel spreadsheet the identification of the borrow sites, needed blending sites, and the options for the location of the soil-blending plants. These inputs are generated during the project planning phase, when the availability of borrow sites with the identification of available materials and volumes, location, and other pertinent data are collected. In reality, a large time gap usually occurs between the conclusion of the design and construction. Often the sites evaluated for material sources and the location of the material blending plants may not be available when construction starts. For these situations, the model provides for an expedited way of evaluating several scenarios.

The determination of the transportation distances for the materials is the most labor-intensive task associated with the proposed methodology. The distances are measured in the plans and manually input in the spreadsheet. However, in the past few years, roadway geometric design has moved towards digital, with computer-aided design (CAD) systems undergoing major developments. The link between CAD roadway design and automation of the inputs to the proposed model documented by this paper has been researched by the authors and is under development.

The unit costs for the materials and labor are required as inputs for the model. The model assumes that the user has access to reliable cost data developed in previous projects. However, the model allows for sensitivity analysis to be performed by simulation of several scenarios for the unit costs.

$L I N G O$ allows for inputs to be prepared using plain text inputs or using a $L I N G O$-specific programming language based on a mathematical formulation. The former option was used in programming the input part in Excel.

With the input data prepared by the user using the Excel spreadsheet, the procedure developed with VBA programmed by the authors prepares a file for $L I N G O$, with the mathematical model defined previously in the paper automating the model set up for $L I N G O$. After $L I N G O$ processes the model, the results are presented in spreadsheet format for the user's ease of interpretation.

In the case studies discussed in this paper, the model results are compared with the original cost estimate for the projects to benchmark the performance of the proposed model.

\section{Case Study 1}

The first case study encompasses the rehabilitation of the CE-176 Highway, in the section linking Aiuaba to Antonina do Norte, whose length is $38.11 \mathrm{~km}$ (23.68 mi). The Department of Buildings, Highways and Transport of Ceará (known as DERT in Brazil) was the responsible organization. The works to be analyzed are on paving (base and subbase) consisting of a thickness of $18 \mathrm{~cm}$ 


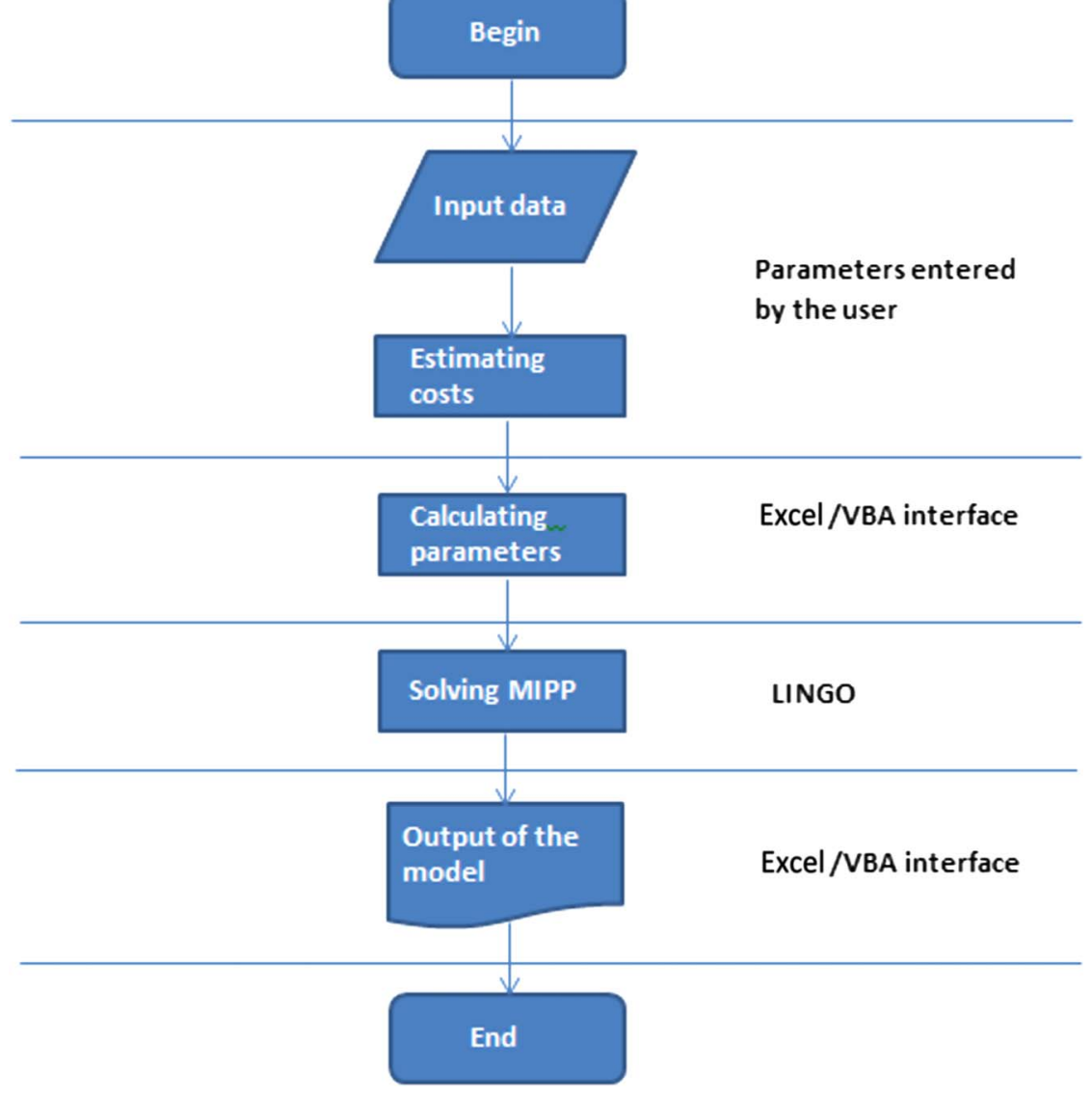

Fig. 4. Flowchart of the proposed methodology

(7.09 in.) base, $17 \mathrm{~cm}$ (6.69 in.) subbase, and double surface treatment of $2.5 \mathrm{~cm}$ ( 1 in.). The pavement layer was not included in the model formulation, given that the small thickness usually needs few sources of aggregates. Therefore, the pavement layer was not included in the mathematical model. In cases where there are a large number of alternative sand, quarries, or positions of the plants, the model can be easily adapted to include the pavement layer.

In this case study, the earthmoving was not analyzed because it consisted only of lateral lending. Based on collected data the problem was modeled by a MIPP solved by LINGO.

The optimal solution indicated that the cost of construction was US $\$ 829,968$. This is the minimum cost for work execution according to the model, and any other viable solution, with the same data, should present a higher cost. The value identified by the model was compared with the cost of the design estimate, so one can calculate how much savings resulted by using the proposed OR approach. However, the design estimate was US\$795,762.

This fact led to a modification of the OR model, given that the design estimate was incompatible with the value found by the initial OR run of US $\$ 829,968$. Since the review of the OR modeling found no errors, the authors investigated the possibility that the solution presented by the design estimate was an infeasible solution, i.e., mathematically impractical.

In this case, the infeasibility may occur due to insufficient material to perform the work. Although there is globally enough material to construct both the base and the subbase, one may identify some feasibility issues in the design estimate. In fact, it was found that the materials of deposits S9 and S12 were not sufficient to implement the project.
According to the manual estimate summarized by the design, the required amount of the S9 deposit material was $11,202,710 \mathrm{~kg}(11,202.71 \mathrm{t})$, but there were only $8,897,700 \mathrm{~kg}$ $(8,897.70 \mathrm{t})$ available, which explains the unfeasibility of the solution. In addition, according to the solution presented by the design, the required amount of the $\mathrm{S} 12$ deposit material was $15,270,060 \mathrm{~kg}(15,270.06 \mathrm{t})$, but only $14,531,400 \mathrm{~kg}$ $(14,531.40 \mathrm{t})$ were available. This was also an unfeasible solution.

The conclusions derived from this case study are that in addition to obtaining the lower-cost solution, the mathematical model showed that the original design estimate for the project was inconsistent.

\section{Case Study 2}

The second case study corresponds to the construction of the CE-176 Highway, in the section linking Arneiroz to Aiuaba, with a length of $31.52 \mathrm{~km}$ (19.59 mi). The agency was the Department of Buildings, Highways and Transport of Ceará (DERT). Based on the collected data, the problem was modeled using a MIPP and processed with the $L I N G O$ solver.

This road section consisted of two strata of pavement: a base and a subbase of stabilized soil, without mixture, and with thicknesses of $18 \mathrm{~cm}$ (7.09 in.) and $17 \mathrm{~cm}$ (6.69 in.), respectively.

This section was subdivided into 122 segments; 61 of these segments were bases and 61 were subbases. To reduce the number of variables, some were considered equal to zero. These variables correspond to deposits that are very distant from the segments and for which the probability of being allocated to the solution were 
low. The problem was then reduced to 798 variables, and the value of the optimal solution corresponded to a total cost of US\$631,869.

Based on the design-estimated budget for the project, it was found that the solution implemented had a cost of US\$657,951, which resulted in $3.92 \%$ savings.

A more detailed analysis of the design estimate showed that, as in the previous case study, the design estimate was a mathematically impractical solution, since the J6 deposit needed 6,140,440 kg $(6,140.44 \mathrm{t})$, when it only had $5,716,650 \mathrm{~kg}(5,716.65 \mathrm{t})$ available.

This shows that the savings resulting by the implementation of the LINGO solutions are probably higher than $3.92 \%$, since the design estimate would have to be corrected to eliminate the infeasible solution.

\section{Case Study 3}

The third case study corresponds to the construction of the CE-085 Highway, in the segment Barrento to Entroncamento CE-176/430 (Aracatiara), with a length of $32.06 \mathrm{~km}$ (19.92 mi). The agency for this section was the Department of Buildings, Highways and Transport of Ceará (DERT). Based on the data collected, the problem was modeled using MIPP and processed using the LINGO solver.

The analyzed construction work was composed of three strata of paving: a base, a subbase, and a segment of reinforced subgrade. The specified thicknesses were a $20-\mathrm{cm}$ (7.87-in.) base and a subbase and reinforced subgrade with variable thicknesses.

The optimal solution value corresponded to a total cost of \$US676,348. The design estimate established a cost of US\$700,695 with the LINGO solution resulting in an economy of $3.47 \%$.

\section{Conclusions}

The model documented in this paper is a powerful tool for allocating earthwork materials, paving materials, and plants along a section of highway in order to obtain the most cost-effective results, considering the constraints inherent to each particular problem.

The innovative approach proposed in this paper is a tool to support decision making in highway construction planning. The model allows the identification of minimum cost solutions in an automated process.

The first author for this paper is a senior engineer with extensive experience in road construction. His experience is incorporated in the proposed methodology, bringing the proposed methodology as close as possible to its application to real-world problems. The case studies and other experimental data gathered during the development of this methodology show that this approach has great potential in providing a robust planning tool for the road construction sector, leading to significant reductions in road construction costs.

The developed approach is not limited to a linear-shaped roadwork or even a roadwork per se. The proposed formulation is quite general and applies to linear sections, constructions with bifurcations, or even large areas of earthmoving. It should be noted that there is a bifurcation in the highway segment presented by Case Study 3. In this case study, the model was applied in the same way and resulted in savings compared with the solution proposed by the design estimate.

The decision to adopt an objective function with separate variables for earthwork and paving improves the solution and provides more flexibility for the user. From the results of Case Study 2, it was possible to easily report the distribution tables of materials for earthwork and paving, allowing for straightforward evaluation of the results. Furthermore, due to the separation of variables, only two constraints were added to the model.
Road projects with small earthmoving quantities or few alternative solutions are unlikely to achieve significant savings by implementation of the modeling techniques summarized by this paper. However, the savings can be significant in road projects with large volumes of earthmoving and a large number of paving mixes.

In the case studies presented, the modeling was implemented based on design estimates, with the objective of comparing results. In practice, it is possible to consider a larger number of alternatives by using the proposed modeling approach, since without the automation provided by the model, the natural tendency is that the number of alternatives to be evaluated manually is reduced to facilitate decision making.

The following are suggestions for future research. The authors are currently pursuing them.

Paving design methods attempt to establish the minimum size for the pavement layers depending on the available materials and the loads applied by the forecasted traffic. However, the minimum thickness identified by the design process is not always the most economical, since the cost depends largely on the total transport distances involved, and if the deposits are at long distances from the roadwork, thinner thicknesses may lead to a higher total cost.

The proposed model of distribution can be applied not only during pavement design and attempts to adjust the thickness of the strata as a function of the structural conditions and traffic, but also to the issues related to other variables such as the type of material.

\section{Notation}

The following symbols are used in this paper:

$C B(i, c a)=$ total unit cost in US\$ $/ \mathrm{m}^{3}$ to run the $c a$ embankment layer with material from the $i$ reservoir (volume of the deposit in situ);

$C D(k, s)=$ total unit cost US\$ $/ \mathrm{m}^{3}$ to have the cutting material from $s$ in the area reserved for $k$ send-off $i$ (volume of the cutting in situ);

$C P X(w p, j)=$ total cost in US\$ to run the $j$ pavement layer, with the mix $w p$ processed on the roadside;

$C S(s, c a)=$ unit cost $\mathrm{US} \$ / \mathrm{m}^{3}$ to run a full layer of $c a$ embankment with material from the layers cutting in (volume of the cutting in situ);

$C Y(w, p, j)=$ total cost in US\$ to run the $j, w$ pavement layer with the mix processed in the manufacturing plant located at position $p$;

caf $=$ number of strata of an embankment;

$F I(i, c a)=$ factor of contraction of the material of the $i$ deposit in relation to the $c a$ embankment layer;

$F S(s, c a)=$ factor of contraction of the $s$ cutting material in relation to the $c a$ embankment layer;

$k f=$ number of areas for send-offs;

$m=$ total number of segments of pavement;

$n=$ total number of deposits;

$p=$ total number of positions available for installation of the manufacturing plant;

$s f=$ number of cutting strata;

$V A(c a)=$ volume in $\mathrm{m}^{3}$ of the $c a$ embankment layer;

$\operatorname{VBF}(k)=$ volume in $\mathrm{m}^{3}$ of the area reserved for the $k$ sendoff;

$V C(s)=$ volume in $\mathrm{m}^{3}$ of the $s$ cut layer;

$\operatorname{VOL}(i)=$ volume in $\mathrm{m}^{3}$ of the $i$ reservoir;

$w f=$ total number of mixes processed in the manufacturing plant;

$w p f=$ total number of mixes processed on the roadside; 


$$
\begin{aligned}
X(w p, j)= & \text { variable decision. The value is equal to } 1 \text { if the } w p \\
& \text { mix processed on the roadside is designated for the } \\
& j \text { segment, and zero otherwise; } \\
X B(i, c a)= & \text { continuous variable. Volume of material to be } \\
& \text { removed from the deposit } i \text { and transported to the } \\
& c a \text { embankment layer, in } \mathrm{m}^{3} \text { (volume of the deposit } \\
& \text { in situ); } \\
X D(k, s)= & \text { continuous variable. Volume of purged material } \\
& \text { layer is disposed in the cut area reserved for } k \text { send- } \\
& \text { off, in } \mathrm{m}^{3} \text { (volume of the cut in situ); } \\
X S(s, c a)= & \text { continuous variable. Volume of material to be } \\
& \text { removed from the cut layer is transported to the } c a \\
& \text { embankment layer, in } \mathrm{m}^{3} \text { (volume of the cut in } \\
& \text { situ); and } \\
& \text { variable decision. The value is equal to } 1 \text { if the } w \\
& \text { mix, processed in the manufacturing plant in } p \\
& \text { position, is assigned to } j \text { segment. The value will be } \\
& \text { zero otherwise. }
\end{aligned}
$$

\section{References}

Christian, J., and Caldera, H. (1988). "Earthmoving cost optimization by operational research." Can. J. Civ. Eng., 15(4), 679-684.

Easa, S. M. (1987). "Earthwork allocations with nonconstant unit costs." J. Constr. Eng. Manage., 113(1), 34-50.
Easa, S. M. (1988). "Earthwork allocations with linear unit costs." J. Constr. Eng. Manage., 114(4), 641-655.

Easa, S. M. (1989). "Earthmoving cost optimization by operational research: Discussion." Can. J. Civ. Eng., 16(3), 408.

Hare, L. W., Koch, V. R., and Lucet, Y. (2011). "Models and algorithms to improve earthwork operations in road design using mixed integer linear programming." Eur. J. Oper. Res., 215(2), 470-480.

Jayawardane, A. K. W., and Price, A. D. F. (1994a). "A new approach for optimizing earth moving operations. Part I." Proc. Instn. Civ. Engrs. Transp., 105(3), 195-207.

Jayawardane, A. K. W., and Price, A. D. F. (1994b). "A new approach for optimizing earth moving operations. Part II.” Proc. ICE Transport., 105(4), 249-258.

LINGO 6 [Computer software]. LINDO Systems 〈www.lindo.com〉.

Mayer, R. H., and Stark, R. M. (1981). "Earthmoving logistics." J. Constr. Div., 107(2), 297-312.

Moselhi, O., and Alshibani, A. (2009). "Optimization of earthmoving operations in heavy civil engineering projects." J. Constr. Eng. Manage., 135(10), 948-954.

Nandgaonkar, S. M. (1981). "Earthwork transportation, allocations: Operations research." J. Constr. Div., 107(1), 373-392.

Ricardo, H. S., and Catalani, G. (2007). Practical guide of excavation: Earthwork and rock excavation (in Portuguese), Pini, São Paulo, Brazil.

Son, J., Mattila, K., and Myers, D. (2005). "Determination of haul distance and direction in mass excavation." J. Constr. Eng. Manage., 131(3), 302-309.

Stark, R. M., and Nicholls, R. L. (1972). Mathematical foundations for design: Civil engineering systems, McGraw-Hill, New York. 